\title{
ESTRATÉGIAS EDUCACIONAIS E SUAS DIFERENTES APLICAÇÕES COMO COMPLEMENTO NA PROMOÇÃO DE UMA EDUCAÇÃO EQUITATIVA
}

Ana L.D.Mateus -dmateus.ana@usp.br

Rua Professor Frederico da Silva Ramos, 298

12600-260 - Lorena - São Paulo

CindyF.Cintra-cindy.cintra@usp.br

Rua Alberto Barbeta, 23, Ap.03

12515-040 - Guaratinguetá - São Paulo

Estela L.Provasi-estelaprovasi@usp.br

Rua Pedro Vicente de Azevedo, 137 - Cruz

12606-050 - Lorena - São Paulo

Pedro H.V. Gentil-pedro.gentil@usp.br

Rua José F de Morais, 37, Ap.04

12602-690 - Lorena - São Paulo

Walton D.de O.Júnior-walton.junior@usp.br

Avenida Papa João XXIII, 228

12604-090 - Lorena - São Paulo

Weberton V. Dias - vinicius.dias.2804@usp.br

Rua Major de Oliveira Borges, 68, Ap. 02

12600-020 - Lorena - São Paulo

USP - Escola de Engenharia de Lorena

Resumo: Ao acompanhar as tendências incorporadas aos novos ciclos educacionais, as estratégias educacionais realçam a importância da experiência para o aprendizado, de modo que a vivência traga a efetividade do que se é chamado de aprender na prática. O objetivo desse artigo é apresentar o desenvolvimento de um projeto de criação de cartilhas contendo estratégias educacionais ativas e reuni-las em um portfólio direcionado a docentes da educação básica. O material foi aplicado em formato de workshop para professores da entidade Marie Curie Vestibulares, demonstrando por meio de questionário avaliativo que o material desenvolvido é uma ferramenta positiva para promoção de uma educação equitativa.

Palavras-chave: Estratégias Educacionais; Role-play; Flipped Classroom; Design Thinking. 


\section{INTRODUÇÃ̃o}

A educação é, desde suas origens, funções e objetivos, um fenômeno social, relacionada assim a diversos contextos coletivos dentro de uma determinada sociedade. $\mathrm{O}$ ato de educar, ainda que seja um processo constante na história, não é o mesmo em todos os tempos, e se encontra vinculado ao projeto de cidadania que se quer ver emergir por meio desse mesmo sistema (DIAS, 2019).

Para Santos (2012), os processos de ensino-aprendizagem necessários à formação educacional encontram-se cada vez mais rediscutidos em virtude das intensas modificações que percorrem o cenário atual brasileiro. Nelas, estão inclusas a velocidade das transformações na sociedade contemporânea, os meios de comunicação na formação do profissional e uma nova configuração de organização do espaço-tempo social. Frente a transformações políticas, culturais e tecnológicas na esfera social contemporânea, surge a exigência de um profissional mais flexível e plurivalente, capaz de atender às demandas dinâmicas do mercado de trabalho do país. Tendo em vista o cenário de adoção quase exclusiva de Metodologias de Ensino Tradicionais por parte da maioria dos educadores ou instituições de ensino, cabe ao setor educacional repensar as práticas empregadas até então.

A divulgação de um conteúdo pronto, por meio da exposição verbal da matéria e exercícios de fixação, torna o aluno uma peça passiva de sua própria aprendizagem. Assim, o processo de ensino atual é um forte candidato à inserção de estratégias educacionais ativas em salas de aula, a fim de oferecer uma formação crítica para futuros profissionais das mais diversas áreas.

No Brasil, um estudo de caso feito pelo Instituto de Ciência e Tecnologia - UNESP demonstra que a não aplicação das metodologias ativas se dá, muitas vezes, pelo desconhecimento das mesmas pela parte dos educadores (BARDINI, 2017).

Nesse contexto, o presente artigo traz como objetivo desenvolver cartilhas contendo estratégias educacionais ativas. O material contém dados relevantes sobre as diferentes formas de ensinar, exemplos práticos e considerações significativas para o funcionamento eficaz da aprendizagem. Inserido como parte de uma capacitação simples, o portfólio se torna uma fonte de informações que, por meio do esclarecimento do conteúdo citado, pretende aumentar o emprego dos métodos ativos em sala de aula.

Este projeto foi desenvolvido na disciplina de Projeto Integrado de Engenharia de Produção I do curso de Engenharia de Produção da Escola de Engenharia de Lorena (USP) por alunos ingressantes do curso no primeiro semestre de 2020.

\section{REVISÃO DE LITERATURA}

Ainda que a proposta de métodos inovadores e ativos de ensino seja antiga (a filosofia socrática já utilizava meios interrogativos em função dos ouvintes), o conceito de metodologia ativa se introduz a partir de 1930 pelo professor inglês R. W. Revans (GARCIA, 2017). Sua definição se pauta em uma série de ações que visam desenvolver a capacidade dos alunos de aprender competências que ainda não possuem (BECK, 2018).

Os princípios de uma metodologia ativa estão relacionados com a personalização da aprendizagem, ou seja, fazer com que o aluno seja parte do processo de ensino da maneira com que este se adeque satisfatoriamente (BECK, 2018). 
A metodologia ativa realça a importância da experiência para o aprendizado, de modo que a vivência traga a efetividade do que se é chamado de aprender na prática. De acordo com Mitre (2008, p. 2135), uma metodologia libertadora torna-se indispensável na "formação de um profissional ativo e apto a aprender a aprender". A agilidade do método se baseia no princípio da autonomia de cada sujeito, reconhecendo que em seu respeito se constrói o melhor método de compreensão do processo de produção.

\subsection{Metodologia de ensino-aprendizagem}

\section{Metodologias tradicionais}

A linha tradicional de ensino originou-se a partir do Iluminismo, no século XVIII, tendo como principal objetivo a universalização do conhecimento. Ela é fundamentada na repartição dos conteúdos em áreas de conhecimento e estas, por sua vez, em disciplinas. A transmissão do conhecimento se dá por um docente e a assimilação é feita de forma passiva pelos discentes. A aquisição do conhecimento é focada na memorização de dados, sem se preocupar com a compreensão destes (HOEVEL, 2018), seguindo uma visão enciclopedista (LACERDA; SANTOS, 2018), sendo averiguado através de avaliações periódicas (PEREIRA, 2019). Desta maneira, o aprendizado é focado no saber do professor (SANTOS et al., 2019) que domina o conteúdo (LACERDA; SANTOS, 2018), enquanto o aluno normalmente aprende através de aulas expositivas, assumindo o papel de aprendiz passivo (PARREIRA, 2018).

O método tradicional de ensino se baseia na ideia de que a inteligência humana apresenta caráter cumulativo, permitindo ao homem armazenar informações complexas ou simples que devem ser adquiridas na instituição escolar, e defende que "para que o estudante seja um sujeito crítico, indagador, independente, ele precisa ter uma base estruturada de informação, atenção e concentração, silêncio e esforço, tendo um ambiente escolar onde se raciocina e que seja convenientemente austero para o aluno não perder a atenção" (PEREIRA, 2019, p. 17). O conteúdo é determinado pela sociedade e legislação, não levando em consideração a experiência do aluno e sua realidade social (PEREIRA, 2019).

\section{Metodologias de aprendizagem ativa}

As metodologias de aprendizagem ativa se fundamentam na escola construtivista (LACERDA; SANTOS, 2018), uma filosofia de ensino inspirada no trabalho do suíço Jean Piaget (1896-1980). O construtivismo se baseia na ideia de que o conhecimento deve ser, como seu próprio nome sugere, construído a partir de situações experimentais, reflexões e da interação e diálogo com os colegas e o professor (PARREIRA, 2018). Cabe ao professor ser mediador do conhecimento (SANTOS, 2015) e espera-se que atue criando condições que permita ao aluno vivenciar situações nas quais os alunos possam construir seu conhecimento.

O aluno assume a posição de protagonista do seu aprendizado (DE SOUZA, 2009; SANTOS et al., 2019) e da aula, contribuindo ativamente para a construção desta e do conhecimento através de pesquisas investigativas, debates e desenvolvimento crítico de conclusões (LACERDA \& SANTOS, 2018).

Diferentemente do modelo tradicional de ensino, a intenção dos modelos ativos é desenvolver a autonomia intelectual dos alunos com atividades que os exigirão sua 
participação ativa em atividades envolvendo pesquisa, reflexão, questionamento, diálogo e resolução de problemas (PEREIRA, 2019).

O uso de metodologias ativas no ensino é comumente embasado por pirâmides ou cones da aprendizagem, as pirâmides geralmente atribuídas a William Glasser, e os cones a Edgar Dale (Figura 1).

Figura 1- Pirâmide da Aprendizagem

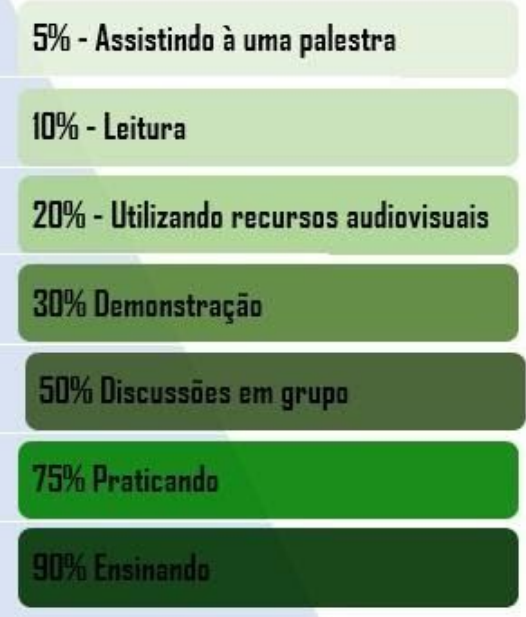

Fonte: Adaptado LASEINDE et al., 2015.

As pirâmides ou cones da aprendizagem pressupõem a existência de uma hierarquia de maneiras de aprendizado (DA SILVA \& MUZARDO, 2018), mostrando um aumento da compreensão dos alunos à medida em que se reduz a necessidade de abstração por parte do aluno.

Analisando a pirâmide de aprendizagem, é possível verificar que métodos que exigem maior participação e menor abstração do aluno se mostram mais eficazes para retenção do conhecimento do que métodos de aprendizado passivos, e que a retenção do conhecimento aumenta gradativamente à medida que os métodos se tornam mais ativos e menos passivos. Sendo assim, atividades como repetir, recordar, demonstrar, explicar ou mesmo falar sobre um assunto se mostram muito eficazes para reter o conhecimento e, consequentemente, aprender sobre dele.

Existem diversas metodologias de aprendizagem ativa, três delas são apresentadas a seguir:

- Role-play: Com o objetivo de auxiliar os alunos a entender e desenvolver habilidades sociais e comportamentos (BILICH \& CIARROCHI, 2009), a aplicação de métodos experienciais que permitem a eles a prática de lidar com problemas da vida real vem sendo cada vez mais aplicados. A estes métodos experienciais, dá-se o nome de role-play. Eles utilizam da dramatização de um cenário específico fornecido aos 
alunos e podem incluir elementos de atuação e improvisação (PISCHETOLA \& MIRANDA, 2019). Após a encenação, os que a executaram pontuam os elementos mais significativos do caso aos demais alunos e ao facilitador da atividade (JALGAONKAR, et al., 2012). Estes, por sua vez, dão seu feedback àqueles que a executaram (BILICH \& CIARROCHI, 2009). Esses métodos contribuem para o aprendizado estimulando os seus sentidos e extrapolando o pensamento racional (PISCHETOLA \& MIRANDA, 2019).

- Flipped Classroom: consiste na inversão do momento e local em que o conhecimento é adquirido, deixando de ocorrer do horário da aula. Desta maneira, os alunos se preparam previamente com um material que cobre o que teria sido ensinado em uma aula tradicional, direcionados pelo professor (ABEYSEKERA \& DAWSON, 2015). A aula se torna o local para resolução de problemas, avanço e desenvolvimento de aprendizado colaborativo (TUCKER, 2012). Após acompanhar a aula, há a consolidação dos conhecimentos (ABEYSEKERA \& DAWSON, 2015). A organização e preparação prévia do aluno contribui para o desenvolvimento de sua autonomia na sua busca individual do conhecimento, assim como elevada didática por parte do professor para criar dinâmicas interativas (PISCHETOLA \& MIRANDA, 2019) e que maximizem os recursos de aprendizagem (TUCKER, 2012), podendo extrapolar a explicação expositiva do conteúdo ao incluir outras dinâmicas como esclarecimento de dúvidas desenvolvimento e desenvolvimento de pensamento crítico (PISCHETOLA \& MIRANDA, 2019);

- Design Thinking: O design thinking surgiu da necessidade econômica de gerar inovação, que por sua vez, é advinda da criatividade em grupo (PISCHETOLA \& MIRANDA, 2019). Porém o conceito evoluiu a partir da necessidade de entender e controlar o processo de design (CALLAHAN, 2019). A maneira de pensar do designer se assemelha a ideação de projetos (PISCHETOLA \& MIRANDA, 2019) e visa atender às necessidades das pessoas com o que é tecnológica e estrategicamente viável e que pode ser convertido em valor para o cliente e em oportunidade de mercado (BROWN, 2008). No contexto educacional, o design thinking se baseia em três pilares: Pare, Pense e Compartilhe. Neste formato, o professor levanta uma questão relevante para os alunos, define um problema/desafio e propõe uma reflexão acerca do assunto, que ocorre inicialmente individual, em seguida em duplas e, por último, é compartilhada com os demais alunos. Uma grande vantagem deste processo é a perspectiva de geração de novas resoluções para a questão proposta (PISCHETOLA \& MIRANDA, 2019).

\section{METODOLOGIA}

As etapas de construção deste artigo (Figura 2), tiveram por objetivo desenvolver uma pesquisa exploratória, sendo iniciada com uma discussão das hipóteses de quais seriam os problemas enfrentados pela educação brasileira para construção de um ensino equitativo, inclusivo e de qualidade.

Foi realizada, em seguida, a pesquisa bibliográfica, a fim de compreender os motivos geradores da problemática. Como resultado compreendeu-se que o uso individualizado de metodologias passivas não possibilita que todos acessem o mesmo nível de educação. Como proposta de solução foi desenvolvido um portfólio para professores, o qual foi transformado em 
um ebook gratuito, definindo o que são Estratégias Educacionais e o passo a passo da execução de algumas de suas vertentes.

Após a finalização do ebook, uma capacitação baseada nesse material foi aplicada, tendo como espaço amostral quinze professores da Marie Curie Vestibulares, entidade social composta em sua maioria por jovens universitários da Escola de Engenharia de Lorena, e a qual tem como objetivo promover o acesso a uma educação de qualidade para jovens em vulnerabilidade socioeconômica.

Finalizada, um questionário foi aplicado com o objetivo de verificar as percepções e possíveis aderências das metodologias em sala de aula.

Figura 2 - Fluxograma das etapas de execução do projeto

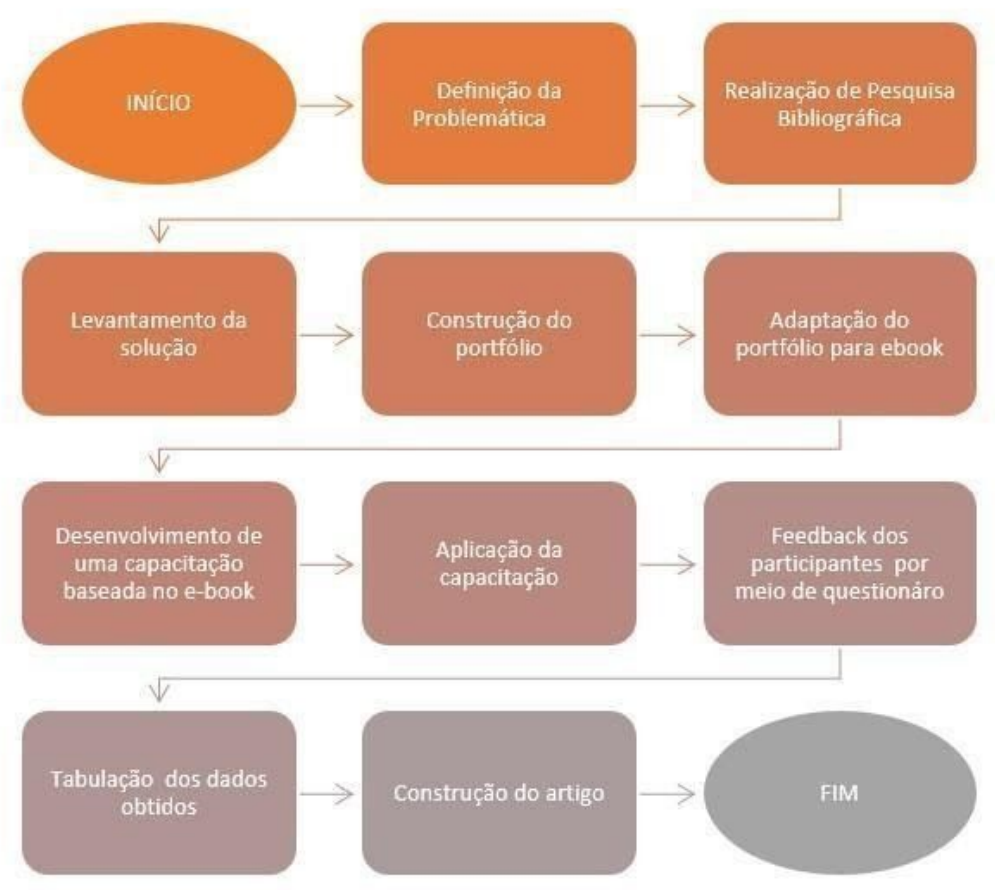

Fonte: Autores (2020)

\section{RESULTADOS E DISCUSSÃO}

Após a criação do Relatório Preliminar e do Portfólio do projeto (presente nas Referências Bibliográficas e disponível em: http://bit.ly/metodologiasativascapacita), foi estruturado um treinamento online de três horas para 15 professores. $\mathrm{O}$ treinamento consistiu em apresentar o funcionamento da metodologia a partir do uso da própria metodologia, o que veio a proporcionar uma experiência nos métodos: Role-Play, Flipped Classroom e Design Thinking. Anterior ao treinamento, os participantes foram introduzidos no assunto por meio da disponibilização de um ebook (Figura 3), contendo conceitos e exemplos práticos a respeito das metodologias o que possibilitou maior assimilação durante a reunião.

Após a finalização do treinamento por vídeo-chamada, cada professor recebeu um certificado simbólico (Figura 4) de agradecimento pela participação. 


\section{COBENGE (C) COBENCE 2020 de Educação em Engenharia
da ABENGE}

Figura 3 - Cartilhas do Portfólio de Estratégias Educacionais Ativas

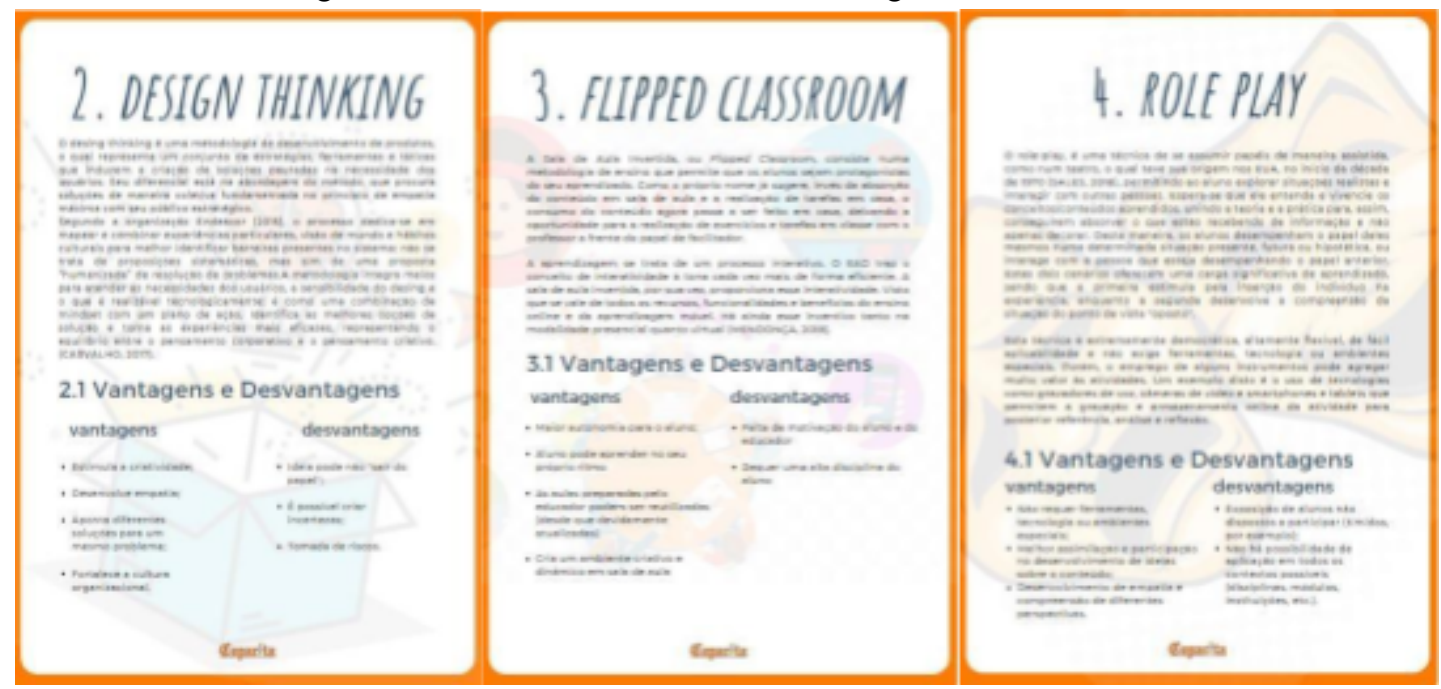

Fonte: Autores (2020)

Figura 4 - Exemplo do certificado de participação para os educadores

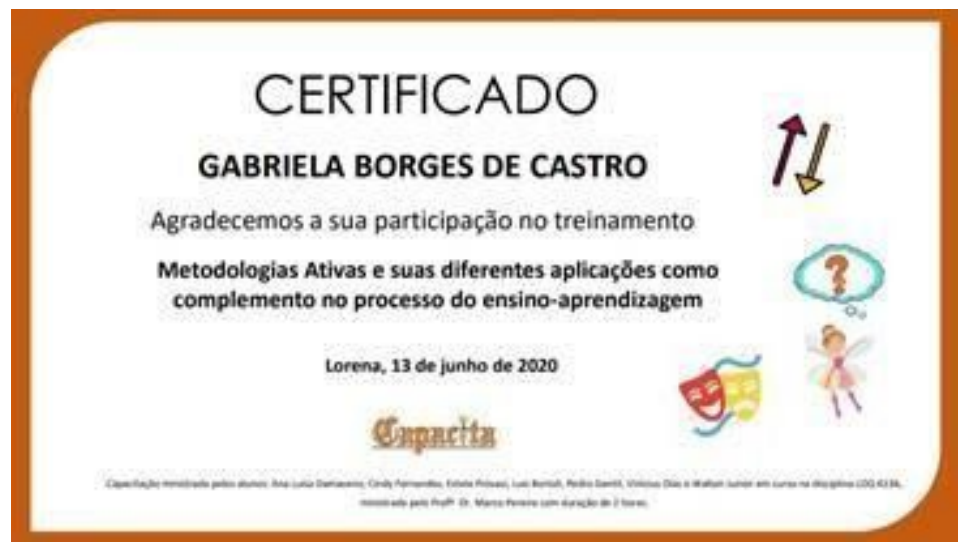

Fonte: Autores (2020)

Segundo os feedbacks, $100 \%$ dos educadores responderam que usariam alguma das metodologias em suas aulas. 100\% aplicariam metodologia Flipped Classroom, 81,8\% aplicariam o Design Thinking e 45,5\% aplicariam o Role-Play. Os professores afirmaram que com a utilização dessas metodologias, o raciocínio dos alunos será melhor estruturado devido ao dinamismo das interações. Também afirmaram que as metodologias permitirão tornar o ambiente de estudo mais desafiador, divertido e lúdico. Essa informação tem impacto no ganho social que é possível ter a partir do conhecimento de uma nova metodologia, que permite complementar os níveis de aprendizado dos alunos. Além das contribuições externas, a construção do Portfólio permitiu a inserção dos integrantes no tema "Educação", o que aumentou a compreensão por parte dos autores sobre a complexidade e dedicação que estão envolvidas por trás da preparação de aulas. Paralelamente, a construção desse material, contribui para a Engenharia de Produção, adentrando o estudante no mundo científico e permitindo despertar seu interesse em pesquisas e na busca pelo conhecimento. 


\section{CONCLUSÃO}

$\mathrm{O}$ artigo apresentou o desenvolvimento de cartilhas esclarecedoras quanto a três estratégias educacionais: Role-play, Design Thinking e Flipped Classroom. O objetivo geral proposto foi concluído, uma vez que houve a reunião do conteúdo exposto em um único portfólio capaz de atingir docentes da rede de educação brasileira, de maneira a contribuir para uma maior aplicação de tais estratégias no país.

Em conjunto com a realização do material, uma capacitação simples foi oferecida a docentes de um cursinho popular situado em Lorena, fato este que permite uma análise de eficácia do conteúdo produzido. Os resultados mostraram que houve grande interesse por parte dos educadores, muitos deles sem conhecimento prévio sobre as estratégias apresentadas, e que o portfólio apresentado pode ser classificado como um conteúdo ativo para a exposição dos métodos educacionais anteriormente citados.

Dessa forma, o presente estudo destaca e estimula à inserção de estratégias ativas de aprendizagem, que carregam consigo mais dinamismo e interações coletivas e que promovem o desenvolvimento de competências transversais tão exigidas no cenário atual do mercado de trabalho brasileiro.

\section{Agradecimentos}

Os autores agradecem ao professor Marco Antonio Carvalho Pereira, ao monitor Lucas Araújo Cavalcanti e à tutora do grupo Ana Luiza Silva pela orientação e participação em todas as etapas do projeto.

\section{REFERÊNCIAS}

ABEYSEKERA, L., \& DAWSON, P. 2015. Motivation and cognitive load in the flipped classroom: definition, rationale and a call for research, Higher Education Research \& Development, 34:1, 1-14.

BARDINI, V. S. dos S., CRUZ, I. D. de S., TANGO, R. N., SPALDING, M. 2017. Levantamento de dados sobre a utilização de metodologias ativas e ferramentas digitais no ensino superior: Estudo de Caso do ICT-UNESP. In.: EDUCERE - XIII Congresso Nacional de Educação, 2017, Curitiba, Paraná. Anais do XIII Congresso... Paraná: PUCPR.

BECK, C. 2018. Metodologias Ativas: conceito e aplicação. Andragogia Brasil. Disponível em: < https://andragogiabrasil.com.br/metodologias-ativas/>. Acesso em 24 mar. 2020.

BILICH, L. L., \& CIARROCHI, J. 2009. Promoting social intelligence using the experiential role-play method. Acceptance and commitment therapy: Contemporary theory research and practice, 247-262.

BROWN, T. 2008. Design thinking. Harvard business review, 86(6), 84.

CALlaHAN, K. C. 2019. Design Thinking in Curricula. The International Encyclopedia of Art and Design Education, 1-6. 
DA SILVA, F. L., \& MUZARDO, F. T. 2018. Pirâmides e cones de aprendizagem: da abstração à hierarquização de estratégias de aprendizagem. Dialogia, (29), 169-179.

DIAS, E, \& PINTO, F.C.F. 2019. Ensaio: Avaliação e Políticas Públicas em Educação. SCIELO, pg 2-3.

DOS SANTOS, C. A. M., PEREIRA, M. A. C., BARRETO, M. A. M., DE SOUZA, M. A., \& CICARELLI, P. O. 2019. CEMTRAL: uma nova metodologia híbrida de ensino e aprendizagem. Revista Brasileira de Aprendizagem Aberta e a Distância, 18(1), 18-18.

GARCIA, André. Blog Unicamp. Aprendizagem ativa é mais antiga do que você imagina. 2017.Disponível

em:

$<$ https://www.blogs.unicamp.br/apedra/2017/10/12/aprendizagem-ativa-emais-antiga-do-quevoce-imagina/>. Acesso em 24 mar. 2020.

HOEVEL, C. 2018. "Seven Myths About Education" de Daisy Christodoulou. Cultura económica, 36(95), 97-99.

JALGAONKAR, S. V., SARKATE, P. V., \& TRIPATHI, R. K. 2012. Students perception about small group teaching techniques: Role play method and case based learning in pharmacology. Education in Medicine Journal, 4(2).

LACERDA, F. C. B., \& SANTOS, L. M. D. 2018. Integralidade na formação do ensino superior: metodologias ativas de aprendizagem. Avaliação: Revista da Avaliação da Educação Superior (Campinas), 23(3), 611-627.

LASEINDE, O. T., ADEJUYIGBE, S. B., MPOFU, K., \& CAMPBELL, H. M. 2015.

Educating tomorrow engineers: Reinforcing engineering concepts through Virtual Reality (VR) teaching aid. In 2015 IEEE International Conference on Industrial Engineering and Engineering Management (IEEM). 1485-1489. IEEE.

MITRE, S.M., SIQUEIRA-BATISTA, R., GIRARDI-de-MENDONÇA, J. M., MORAiSPINTO, N. M. D., MEIRELlES, C. D. A. B., PINTO-PORTO, C., MOREIRA, T., \& Hoffmann, L. M. A. 2008. Metodologias ativas de ensino-aprendizagem na formação profissional em saúde: debates atuais. Ciência \& saúde coletiva, 13, 2133-2144.

PARREIRA, J. E. 2018. Aplicação e Avaliação de uma Metodologia de Aprendizagem Ativa (tipo ISLE) em Aulas de Mecânica, em cursos de Engenharia. Revista Brasileira de Ensino de Física, 40(1), e1401.

PEREIRA, E. V. 2019. A importância das metodologias ativas e método tradicional para o processo de ensino e aprendizagem no ensino fundamental. Trabalho de Conclusão de Curso - Faculdades Anhanguera, Itapecerica da Serra, SP. 
PISCHETOLA, M., \& MIRANDA, L. T. D. 2019. Metodologias ativas, uma solução simples para um problema complexo. Revista Educação e Cultura Contemporânea, 16(43), 30-56. SANTOS, G. 2012. A educação e as transformações na sociedade. In: Brasil Escola, Educação, p. 4-6.

TUCKER, B. 2012. The flipped classroom. Education next, 12(1), 82-83.

GRUPO CAPACITA. Estratégias Educacionais e suas diferentes aplicações como complemento na promoção de uma educação equitativa. Disponível em: http://bit.ly/metodologiasativascapacita.

\section{EDUCATIONAL STRATEGIES AND THEIR DIFFERENT APPLICATIONS AS A COMPLEMENT IN PROMOTING EQUITABLE EDUCATION}

Abstract: By following the trends incorporated into the new educational cycles, educational strategies emphasize the importance of experience for learning, so that the experience brings the effectiveness of what is called learning in practice. The objective of this article is to introduce the development of a project to create booklets containing active educational strategies and bring them together in a portfolio aimed at basic education teachers. The material was applied in the format of a workshop for teachers from the Marie Curie Vestibulares entity, demonstrating through an evaluative questionnaire that the material developed is a positive tool for promoting equitable education.

Keywords: Educational Strategies; Role-play; Flipped Classroom; Design Thinking. 